\title{
On Structure Descriptors Related with Intramolecular Energy of Alkanes
}

\author{
Ivan Gutman, Boris Furtula, and Biljana Arsića \\ Faculty of Science, University of Kragujevac, P. O. Box 60, 34000 Kragujevac, Serbia and \\ Montenegro, and \\ ${ }^{a}$ Faculty of Science, University of Niš, Višegradska 33, 18000 Niš, Serbia and Montenegro \\ Reprint requests to Prof. I. G.; Fax: +381 34 335040; E-mail: gutman@knez.uis.kg.ac.yu
}

Z. Naturforsch. 59a, $694-698$ (2004); received June 8, 2004

In an earlier work it was demonstrated that the Zenkevich index $U$ provides a measure of the intramolecular energy of an organic molecule, and that - in the case of alkanes - it is related to the Wiener index. We now show that $U$ is much closer related to the recently introduced variable Wiener index $W_{\lambda}$ : Within sets of isomeric alkanes, the relation between $U$ and $W_{\lambda}$ is linear, the $\left(U, W_{\lambda}\right)$-points forming several, mutually parallel, lines. Each such line pertains to a group of isomers possessing a fixed number of methyl groups. There exists a critical value of the parameter $\lambda$ for which all the $\left(U, W_{\lambda}\right)$-lines coalesce, in which case the relation between $U$ and $W_{\lambda}$ becomes independent of the number of methyl groups. Approximate analytical expressions for the $\left(U, W_{\lambda}\right)$-dependence are deduced.

Key words: Wiener Index; Variable Wiener Index; Zenkevich Index; Intramolecular Energy; Alkanes.

\section{Introduction}

In a recent paper [1] it was established that in the case of alkanes, the structure-descriptor $U$, introduced a short time ago by Zenkevich [2-6], and the classical Wiener index $W$ [7-10] are mutually related. From a physico-chemical point of view this is a surprising finding, because $U$ is conceived as a (rough) measure of the intramolecular energy, whereas $W$ has been recognized as a (rough) measure of the molecular surface area [11] and intermolecular van der Waals forces [12]. The reason for the existence of a relation between $U$ and $W$ lies in the fact that both quantities depend on the same structural features of the underlying molecular graph [1]. More specifically, if $T$ is the graph representing an alkane molecule $\mathrm{C}_{n} \mathrm{H}_{2 n+2}$ (in which case $T$ necessarily is a tree [13]), then

$$
W=\sum_{e} n_{1}(e \mid T) n_{2}(e \mid T)
$$

and

$$
U=\sum_{e} \sqrt{\frac{(C+2 H) n+2 H}{\left[(C+2 H) n_{1}(e \mid T)+H\right]\left[(C+2 H) n_{2}(e \mid T)+H\right]}},
$$

where $n$ is the number of vertices of $T$, and where $H$ and $C$ stand for the atomic masses of hydrogen and carbon $(H=1.0, C=12.0)$.
In (1) and (2), $n_{1}(e \mid T)$ and $n_{2}(e \mid T)$ denote the number of vertices lying on the two sides of an edges $e$ of $T$, whereas the summations go over all edges of the molecular graph $T$. Recall that an $n$-vertex tree possesses $n-1$ edges, and for each of these edges,

$$
n_{1}(e \mid T)+n_{2}(e \mid T)=n .
$$

A modification of the Wiener index was recently proposed [14-16], viz.,

$$
W_{\lambda}=\sum_{e}\left[n_{1}(e \mid T) n_{2}(e \mid T)\right]^{\lambda},
$$

where $\lambda$ is an adjustable parameter. The structuredescriptor $W_{\lambda}$ was named [15] "variable Wiener index". Clearly, for $\lambda=1$ the variable Wiener index reduces to the ordinary Wiener index.

By comparing the right-hand sides of (1), (2), and (4), we may expect that for some values of the parameter $\lambda$, different from unity, the (approximate) relation between $U$ and $W_{\lambda}$ will become much more accurate than between $U$ and $W$. As shown below, this indeed is the case.

\section{The Methyl Group Rule}

Initially it was believed [14] that the combination of (2) and (4) implies the existence of a linear correlation 

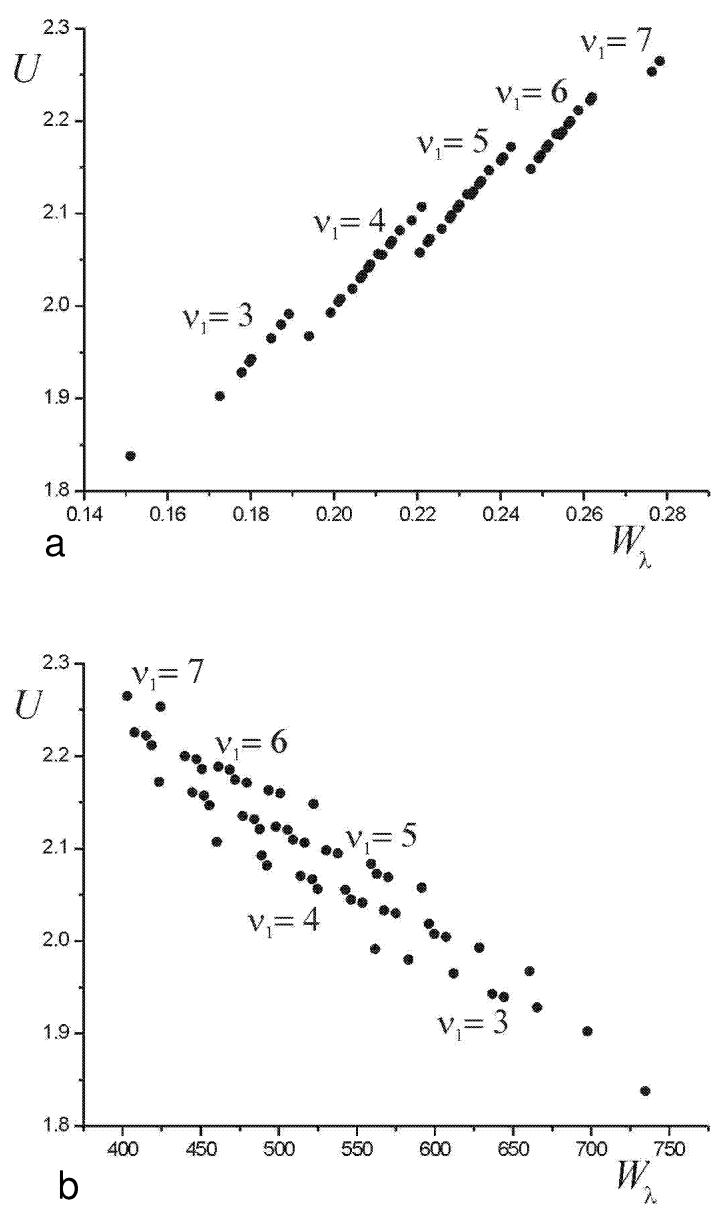

Fig. 1. The Zenkevich index $(U)$ versus the variable Wiener index $\left(W_{\lambda}\right)$ for isomeric decanes $(n=10)$, for (a) $\lambda=-1.5$ and (b) $\lambda=+1.5$. The data-points lie on several lines, each line corresponding to isomers with a fixed number $(v)$ of methyl-groups; the "outlyer" is the data-point corresponding to $n$-decane, the only isomer with two methyl groups. Note that the ordering of these lines (with regard to $v$ ) in diagram (a) is opposite to that in diagram (b). This inversion pertains to the sign-change of the coefficient $\gamma$ in (5).

between $U$ and $W_{\lambda}$. However, the actual relation between $U$ and $W_{\lambda}$ is somewhat more complicated. If $n$ is sufficiently large (say, $n \geq 10$ ), then the $\left(U, W_{\lambda}\right)$-points form a pattern illustrated in Figure 1.

As seen from Fig. 1, the $\left(U, W_{\lambda}\right)$-points form several lines. These are nearly parallel and nearly equidistant. The clue for rationalizing such a behavior was the observation that the points belonging to a line correspond to alkane isomers with the same number $v$ of methyl groups. In other words, the respective molecular graphs have the same number of vertices of degree one.
That this is a generally applicable regularity was checked in the case of $\lambda=1$ by testing all alkanes with up to $n=15$ carbon atoms [17]. The analysis was then repeated for various values of $\lambda$ in the interval $[-10,+10]$; as explained below, the case $\lambda=0$ is exceptional and was therefore avoided. It was found that, for a fixed value of $n$, the slopes of the regression lines are remarkably similar, implying that these lines are almost parallel. Their distances vary with increasing values of $v$, and depend very much on $\lambda$. The analysis of the computed data reveals that the claim that the $(U, W)$-lines are equidistant is acceptable only as a plausible, but not very accurate approximation (cf. Fig. 1).

Anyway, the high correlation coefficients, obtained for all examined values of $n$ and $v$, and for $-10 \leq \lambda \leq$ +10 , confirm that it is justified to separately consider the data points for fixed $n$ and $v$, that within each of such group of data points, the Zenkevich and the variable Wiener index are linearly correlated, and that the number of methyl groups is the most important structural parameter that, in addition to $n$, influences the relation between $U$ and $W_{\lambda}$.

By the above outlined empirical examination of the relations between the Zenkevich and the variable Wiener indices, the following regularity could be established, that we refer to as the

Methyl Group Rule: For a fixed value of $\lambda \neq 0$, the $\left(U, W_{\lambda}\right)$-points lying (approximately) on the same line pertain to isomeric alkanes with equal number of methyl groups; the $\left(U, W_{\lambda}\right)$-points corresponding to isomeric alkanes with different number of methyl groups lie on different lines.

The way in which the pattern shown in Fig. 1 changes with $\lambda$ is essentially the following:

For $\lambda>0$ the Zenkevich index is a decreasing function of $W_{\lambda}$. As $\lambda$ decreases, the respective regression lines become steeper, and their slopes tend to $-\infty$ when $\lambda \rightarrow 0$. For $\lambda<0$ the Zenkevich index is an increasing function of $W_{\lambda}$. As $\lambda$ increases, the respective regression lines become steeper, and their slopes tend to $+\infty$ when $\lambda \rightarrow 0$. There exists a critical value of $\lambda$, denoted by $\lambda_{0}$, at which the regression lines pertaining to various values of $v$ coalesce. For $\lambda>\lambda_{0}$ the line for $v=k+1$ lies above the line for $v=k$. For $\lambda<\lambda_{0}$ the line for $v=k+1$ lies below the line for $v=k$.

This relatively complicated behavior of the relation between $U$ and $W_{\lambda}$ could be rationalized by a pertinent mathematical analysis of (2). As shown below, 
the Methyl Group Rule and its dependence on $\lambda$ are reasonably well reproduced by the approximation (5):

$$
U \approx-\alpha W_{\lambda}+\beta+\gamma v
$$

where the coefficients $\alpha, \beta$, and $\gamma$ are functions of the variable $\lambda$, and, in addition, depend only on $n$.

According to (5), for a given value of $n$, the $\left(U, W_{\lambda}\right)$ points lie on several mutually parallel lines (all having the same slope $-\alpha$ ). The relative position of these lines depends on the sign of $\gamma$, as shown in Figure 1. The way in which $\alpha, \beta$, and $\gamma$ change with $\lambda$ is seen from Figs. 2, 3, and 4, and is discussed in due detail below.

\section{Analysis of the Relation between $U$ and $W_{\lambda}$}

Introduce first an auxiliary variable $x$

$$
x(e)=\left[n_{1}(e \mid T) n_{2}(e \mid T)\right]^{\lambda} .
$$

Then, bearing in mind (3), we can rewrite (2) as

$$
U=A \sum_{e}\left(x(e)^{1 / \lambda}+B\right)^{-1 / 2}
$$

where

$$
A=\frac{\sqrt{(C+2 H) n+2 H}}{C+2 H}
$$

and

$$
B=\frac{H n}{C+2 H}+\left(\frac{H}{C+2 H}\right)^{2} .
$$

Note that both $A$ and $B$ depend only on the number $n$ of carbon atoms, and are independent of any other structural detail of the alkane molecule under consideration.

The summation on the right-hand side of (7) goes over all edges of the respective molecular graph, i. e., over all carbon-carbon bonds. Bearing in mind the Methyl Group Rule outlined in the previous section, this sum is now divided into two parts: one pertaining to the edges whose one endpoint is a vertex of degree one, and the other embracing all other edges. There are $v$ edges whose one endpoint is a vertex of degree one. For these edges, $n_{1}=1, n_{2}=n-1$, and thus $x(e)=(n-1)^{\lambda}$. Consequently

$$
U=A v(n-1+B)^{-1 / 2}+A \sum_{*}\left(x(e)^{1 / \lambda}+B\right)^{-1 / 2},
$$

where $\sum_{*}$ indicates summation over edges of the molecular graph connecting vertices whose degrees are greater than one. There exist $n-1-v$ such edges. For these edges, the product $n_{1} n_{2}$ assumes values from the interval $\left[2 n-4,\left\lfloor n^{2} / 4\right\rfloor\right]$. Therefore the variable $x(e)$, defined by (6), belongs either to the interval $\left[(2 n-4)^{\lambda},\left(\left\lfloor n^{2} / 4\right\rfloor\right)^{\lambda}\right]$ if $\lambda \geq 0$, or to the interval $\left[\left(\left\lfloor n^{2} / 4\right\rfloor\right)^{\lambda},(2 n-4)^{\lambda}\right]$ if $\lambda \leq 0$. For any value of $\lambda$ the center of this interval is at

$$
\xi=\frac{1}{2}\left[(2 n-4)^{\lambda}+\left(\left\lfloor n^{2} / 4\right\rfloor\right)^{\lambda}\right] .
$$

In view of (7) and (10), and taking into account (4), we consider the function $\left(x^{1 / \lambda}+B\right)^{-1 / 2}$ and expand it into a power series in the variable $x$, around the point $\xi$. By direct calculation one gets

$$
\begin{aligned}
& \left(x^{1 / \lambda}+B\right)^{-1 / 2}= \\
& \left(\xi^{1 / \lambda}+B\right)^{-1 / 2}-\frac{1}{2 \lambda}\left(\xi^{1 / \lambda}+B\right)^{-3 / 2} \xi^{1 / \lambda-1}(x-\xi)
\end{aligned}
$$

+ higher order terms.

Neglecting the higher order terms, proportional to $(x-\xi)^{2},(x-\xi)^{3}$, etc, we arrive at

$$
\begin{aligned}
U \approx & A v(n-1+B)^{-1 / 2} \\
& +A \sum_{*}\left[\left(\xi^{1 / \lambda}+B\right)^{-1 / 2}+\frac{1}{2 \lambda}\left(\xi^{1 / \lambda}+B\right)^{-3 / 2}\right. \\
& \left.\quad-\frac{1}{2 \lambda}\left(\xi^{1 / \lambda}+B\right)^{-3 / 2} \xi^{1 / \lambda-1} x(e)\right] \\
= & A v(n-1+B)^{-1 / 2}+A(n-1-v) \\
& \cdot\left[\left(\xi^{1 / \lambda}+B\right)^{-1 / 2}+\frac{1}{2 \lambda}\left(\xi^{1 / \lambda}+B\right)^{-3 / 2}\right] \\
& -\frac{A}{2 \lambda}\left(\xi^{1 / \lambda}+B\right)^{-3 / 2} \xi^{1 / \lambda-1} \sum_{*} x(e) .
\end{aligned}
$$

Because of

$$
\sum_{e} x(e)=v(n-1)^{\lambda}+\sum_{*} x(e),
$$

from (4) we get

$$
\sum_{*} x(e)=W_{\lambda}-v(n-1)^{\lambda} .
$$

When the latter relation is substituted back into (12), we obtain the following expressions for the coefficients occurring on the right-hand side of the Ansatz (5):

$$
\alpha=\alpha(\lambda)=\frac{A \xi^{1 / \lambda-1}}{2 \lambda}\left(\xi^{1 / \lambda}+B\right)^{-3 / 2}
$$




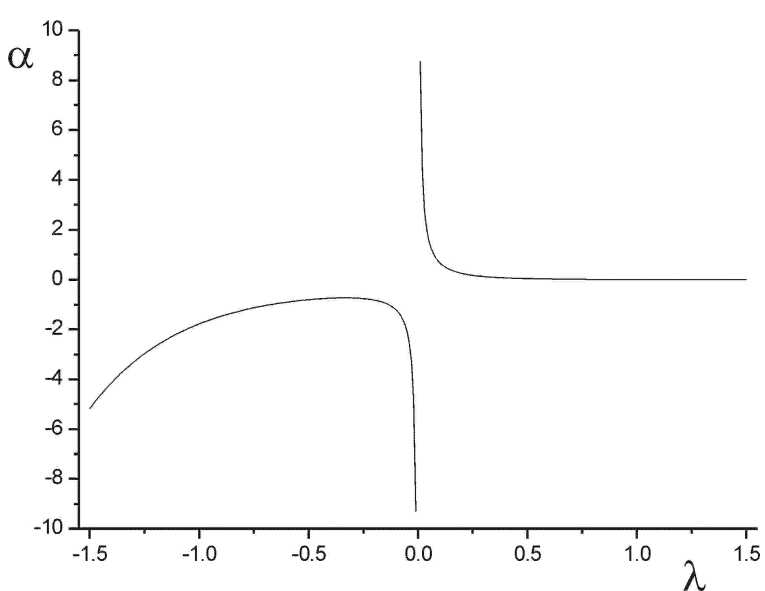

Fig. 2. Dependence of the coefficient $\alpha$ in (5) on the parameter $\lambda$, in the (typical) case of $n=10$.

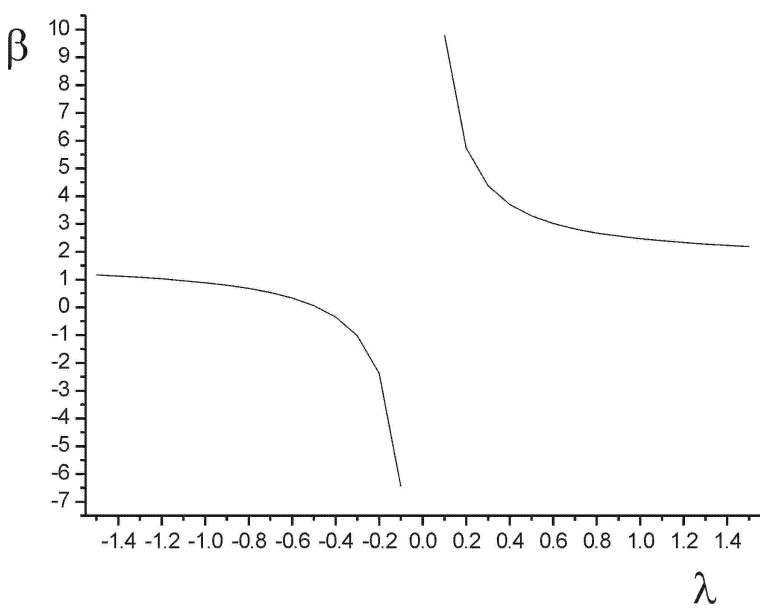

Fig. 3. Dependence of the coefficient $\beta$ in (5) on the parameter $\lambda$, in the (typical) case of $n=10$.

$$
\begin{aligned}
\beta & =\beta(\lambda) \\
= & A(n-1)\left[\left(\xi^{\frac{1}{\lambda}}+B\right)^{-\frac{1}{2}}+\frac{\xi^{\frac{1}{\lambda}}}{2 \lambda}\left(\xi^{\frac{1}{\lambda}}+B\right)^{-\frac{3}{2}}\right], \\
\gamma= & \gamma(\lambda) \\
= & A\left\{(n-1+B)^{-\frac{1}{2}}-\left(\xi^{\frac{1}{\lambda}}+B\right)^{-\frac{1}{2}}\right. \\
& \left.\quad+\frac{\xi^{\frac{1}{\lambda}}-1}{2 \lambda}\left(\xi^{\frac{1}{\lambda}}+B\right)^{-\frac{3}{2}}\left[(n-1)^{\lambda}-\xi\right]\right\} .
\end{aligned}
$$

Recall that $A, B$, and $\xi$, defined via (8), (9), and (11), are constants, independent of $\lambda$ and dependent only on the number $n$ of carbon atoms in the alkane molecule considered. Therefore, the coefficients $\alpha, \beta$, and $\gamma$ in (5) can be viewed as functions (having a rather compli-
Table 1 . The critical value of the parameter $\lambda$, denoted by $\lambda_{0}$ and obtained by solving the equation $\gamma(\lambda)=0$, with $\gamma(\lambda)$ given by (15), as well as the coefficients $\alpha_{0}$ and $\beta_{0}$ occurring in (16); for details see text.

\begin{tabular}{rccc}
\hline$n$ & $\lambda_{0}$ & $\alpha_{0}$ & $\beta_{0}$ \\
\hline 7 & -0.419 & -0.65615 & -0.17681 \\
8 & -0.424 & -0.69286 & -0.18546 \\
9 & -0.428 & -0.72798 & -0.19426 \\
10 & -0.431 & -0.76159 & -0.20308 \\
11 & -0.433 & -0.79384 & -0.21187 \\
12 & -0.435 & -0.82484 & -0.22059 \\
13 & -0.437 & -0.85471 & -0.22926 \\
14 & -0.439 & -0.88356 & -0.23783 \\
15 & -0.440 & -0.91146 & -0.24635 \\
16 & -0.441 & -0.93851 & -0.25478 \\
17 & -0.442 & -0.96478 & -0.26309 \\
18 & -0.443 & -0.99030 & -0.27142 \\
19 & -0.444 & -1.01517 & -0.27962 \\
20 & -0.445 & -1.03940 & -0.28777 \\
21 & -0.446 & -1.06305 & -0.29589 \\
22 & -0.446 & -1.08616 & -0.30389 \\
23 & -0.447 & -1.10875 & -0.31190 \\
24 & -0.447 & -1.13087 & -0.31982 \\
25 & -0.448 & -1.15253 & -0.32769 \\
26 & -0.449 & -1.17379 & -0.33546 \\
27 & -0.449 & -1.19462 & -0.34326 \\
28 & -0.449 & -1.21507 & -0.35103 \\
29 & -0.450 & -1.23517 & -0.35870 \\
30 & -0.450 & -1.25491 & -0.36639 \\
\hline
\end{tabular}

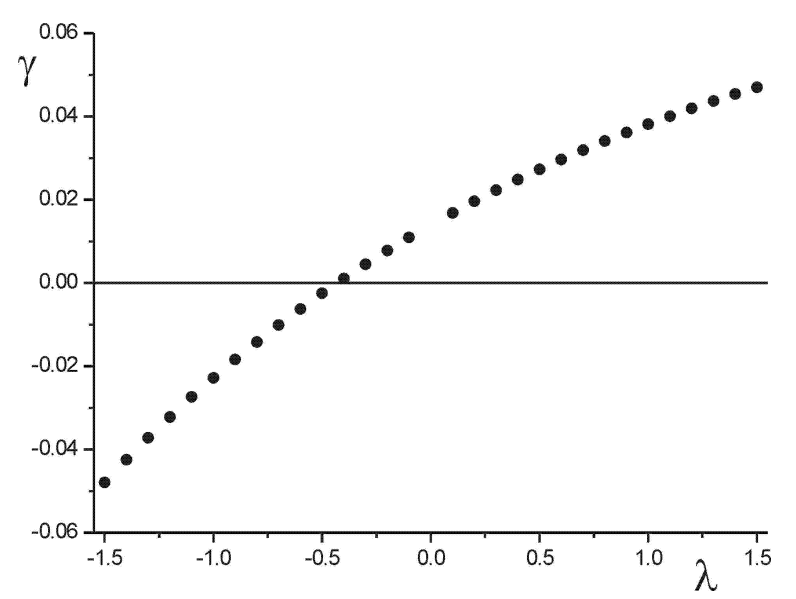

Fig. 4. Dependence of the coefficient $\gamma$ in (5) on the parameter $\lambda$, in the (typical) case of $n=10$. At $\lambda=-0.43$ this coefficient changes sign, resulting in the inversion shown in Fig. 1; for details see Table 1.

cated algebraic form) of the variable $\lambda$, dependent, in addition, on the parameter $n$. The $\lambda$-dependence of $\alpha$, $\beta$, and $\gamma$ is illustrated in Figs. 2, 3, and 4, for decanes $(n=10)$. 


\section{Discussion}

In order to understand the $\lambda$-dependence of $\alpha$ and $\beta$ in (5), one should note that for $\lambda=0$ the variable Wiener index of all alkanes $C_{n} H_{2 n+2}$ is equal to $n-1$. Therefore, when the Zenkevich index is plotted versus the variable Wiener index at $\lambda=0$, a vertical line is obtained. In other words, the coefficients $\alpha$ and $\beta$, viewed as functions of $\lambda$, possess a singularity at $\lambda=0$. This feature is well reproduced by the expressions (13) and (14), cf. Figs. 2 and 3.

As seen from the examples given in Fig. 1, the mutual position of the $\left(U, W_{\lambda}\right)$-lines (with regard to their $\nu$-values) differs for different values of $\lambda$. The type of the ordering of these lines depends on the sign of the coefficient $\gamma$ in (5). As seen from Fig. 4, at a certain value of $\lambda$, the function $\gamma=\gamma(\lambda)$ changes sign, i. e., it is equal to zero. This "critical" value is denoted by $\lambda_{0}$. For $\lambda \approx \lambda_{0}$, the relation between the Zenkevich and the variable Wiener indices becomes independent of the number of methyl groups. In other words, at $\lambda \approx \lambda_{0}$

[1] I. Gutman and I. G. Zenkevich, Z. Naturforsch. 57a, 824 (2002).

[2] I. G. Zenkevich, Fresenius J. Anal. Chem. 365, 305 (1999).

[3] I. G. Zenkevich, Rus. J. Phys. Chem. 73, 797 (1999).

[4] I. G. Zenkevich, Zh. Anal. Khim. 55, 1091 (2000).

[5] I. G. Zenkevich, Zh. Org. Khim. 37, 283 (2001).

[6] I. G. Zenkevich and A.N. Marinichev, Zh. Struct. Khim. 42, 893 (2001).

[7] H. Wiener, J. Amer. Chem. Soc. 69, 17 (1947).

[8] D. H. Rouvray and W. Tatong, Z. Naturforsch. 41a, 1238 (1986)

[9] R. Todeschini and V. Consonni, Handbook of Molecular Descriptors, Wiley-VCh, Weinheim 2000.

[10] A. A. Dobrynin, R. Entringer, and I. Gutman, Acta Appl. Math. 66, 211 (2001). the $\left(U, W_{\lambda}\right)$-lines, pertaining to different values of $v$, coalesce. Then (and only then) a simple linear correlation between $U$ and $W_{\lambda}$ can be observed [14].

Thus, our analysis reveals that the relation between the Zenkevich and the variable Wiener indices should be best considered when $\lambda=\lambda_{0}$. In that case this relation is simplified to:

$$
U \approx-\alpha_{0} W_{\lambda_{0}}+\beta_{0}
$$

where $\alpha_{0}=\alpha\left(\lambda_{0}\right)$ and $\beta_{0}=\beta\left(\lambda_{0}\right)$.

The values of $\lambda_{0}, \alpha_{0}$, and $\beta_{0}$ for the chemically relevant values of $n$ are given in Table 1 .

The definition of the variable Wiener index, (4), is much simpler than that of the Zenkevich index, (2). Consequently, the dependence of $W_{\lambda}$ on the molecular structure is easier to envisage than the analogous dependence of $U$. In view of this, (5) and, especially, (16) make the study of the intramolecular (mainly vibrational) energy of alkanes, and its dependence on the molecular structure, somewhat easier task.

[11] I. Gutman and T. Körtvélyesi, Z. Naturforsch. 50a, 669 (1995).

[12] I. Gutman and J. H. Potgieter, J. Serb. Chem. Soc. 62, 185 (1997).

[13] M. Fischermann, I. Gutman, A. Hoffmann, D. Rautenbach, D. Vidović, and L. Volkmann, Z. Naturforsch. 57a, 49 (2002).

[14] I. Gutman, D. Vidović, B. Furtula, and I. G. Zenkevich, J. Serb. Chem. Soc. 68, 401 (2003).

[15] B. Lučić, A. Miličević, S. Nikolić, and N. Trinajstić, Indian J. Chem. 42A, 1279 (2003).

[16] I. Gutman, D. Vukičević, and J. Žerovnik, Croat. Chem. Acta 77, 103 (2004).

[17] I. Gutman, B. Furtula, B. Arsić, and Ž. Bošković, J. Serb. Chem. Soc. 69, 265 (2004). 